\title{
Insulin Autoantibody Isotypes during the Prediabetic Process in Young Children with Increased Genetic Risk of Type 1 Diabetes
}

\author{
SANNA HOPPU, MATTI S. RONKAINEN, TEIJA KIMPIMÄKI, SATU SIMELL, SARI KORHONEN, \\ JORMA ILONEN, OLLI SIMELL, AND MIKAEL KNIP \\ Juvenile Diabetes Research Foundation Center for Prevention of Type 1 Diabetes in Finland including \\ Pediatric Research Center [S.H., T.K.], University of Tampere Medical School, FIN-33014, Tampere, Finland \\ and Department of Pediatrics [M.K.], Tampere University Hospital, FIN-33521 Tampere, Finland; Hospital for \\ Children and Adolescents [M.K.], University of Helsinki, FIN-00029 Helsinki, Finland; Departments of \\ Pediatrics [S.S., O.S.] and Virology [J.I.], University of Turku, FIN-20520 Turku, Finland; and Department of \\ Pediatrics [M.S.R., S.K.], University of Oulu, FIN-90014 Oulu, Finland
}

\begin{abstract}
ABST
This work aimed to assess the maturation of the humoral
immune response to insulin in preclinical type 1 diabetes by
observing the emergence of various isotypes of insulin autoan-
tibodies (IAA) in children with HLA-DQB1-conferred disease
susceptibility. The series was derived from the Finnish Type 1
Diabetes Prediction and Prevention Study and comprised 15
IAA-positive children who presented with type 1 diabetes during
prospective observation (progressors) and 30 children who re-
mained nondiabetic (nonprogressors). An isotype-specific radio-
binding assay was used to determine isotype-specific IAA
(IgG1-4 and IgA) from samples obtained with an interval of 3-12
mo. The progressors had IAA of subclass IgG3 in their first
IAA-positive sample more often than did the nonprogressors (13
of 15 versus 12 of $30 ; p=0.003$ ). Nine progressors had a
dominant IgG1-IAA response initially, and six had a dominant
IgG3-IAA response. The corresponding distribution among the
nonprogressors was that 20 had a dominant IgG1-IAA response,
none had an IgG3-IAA response, and three had a dominant
response other than IgG1- or IgG3-IAA $\left(\chi^{2}\right.$ df $=2=12.02 ; p=$
0.002). The progressors had higher integrated levels (area under
the curve) of IgG1-IAA ( $p=0.05$ ) and IgG3-IAA ( $p=0.002$ ).
\end{abstract}
Type 1 diabetes is perceived as a chronic autoimmune disease that is thought to result from $\mathrm{T}$ cell-mediated destruction of the insulin-producing pancreatic $\beta$ cells (1). During an

Received February 18, 2003; accepted July 10, 2003.

Correspondence: Mikael Knip, M.D., Ph.D., Hospital for Children and Adolescents, University of Helsinki, P.O. Box 281, FIN-00029 HUCH, Helsinki, Finland; e-mail mikael.knip@hus.fi

This work and the DIPP study have been supported by the Juvenile Diabetes Research Foundation International (grants 197032 and 4-1998-274); the Medical Research Funds, Oulu; Tampere and Turku University Hospitals; the Research Council for Health; Academy of Finland; the Diabetes Research Foundation in Finland; the Päivikki and Sakari Sohlberg Foundation, Helsinki, Finland; the Novo Nordisk Foundation; and the EU Biomed 2 grant (BMH4-CT98-3314).

DOI: 10.1203/01.PDR.0000100905.41131.3F
Nine progressors had a dominant integrated IgG1-IAA response and six had a dominant IgG3-IAA response over the observation period, whereas 22 nonprogressors had a dominant IgG1-IAA response, six had a dominant IgG2-IAA response, and one an IgG3-IAA response $\left(\chi_{\mathrm{df}=2}^{2}=11.23 ; p=0.004\right)$. Genetically susceptible young children who progress rapidly to clinical type 1 diabetes are characterized by strong $\operatorname{IgG} 1$ and $\mathrm{IgG} 3$ responses to insulin, whereas a weak or absent IgG3 response is associated with relative protection from disease. (Pediatr Res 55: 236-242, 2004)

$\quad$ Abbreviations
AUC, area under the curve
GAD65, 65-kD isoform of glutamic acid decarboxylase
IAA, insulin autoantibodies
ICA, islet cell antibodies
INF- $\gamma$, interferon- $\gamma$
RU, relative units
SDS, SD score
Th1/Th2, T-helper $1 /$ T-helper 2
TNF- $\alpha$, tumor necrosis factor- $\alpha$

asymptomatic destructive process of variable duration, various autoantibodies to islet cell autoantigens can be detected, and these are useful for the identification of individuals with an increased risk of clinical disease (2). Several antigens have been observed to be targets of humoral autoimmunity, the three major ones being the $65-\mathrm{kD}$ isoform of glutamic acid decarboxylase (GAD65), the protein tyrosine phosphatase-related IA-2 protein, and insulin, the last being the only truly $\beta$ cell-specific antigen. Insulin autoantibodies (IAA) are often the first autoantibodies to appear during the prediabetic phase (3-5), and they are usually detectable in young children at the diagnosis of type 1 diabetes (3-7). IAA levels correlate in- 
versely with age, the highest levels being seen in children who present with diabetes under the age of $5 \mathrm{y}(7)$.

The isotype profile of antigen-specific autoantibodies may reflect the T-helper 1/T-helper 2 (Th1/Th2) balance of immunity (8), which can change during the prediabetic period. It has been speculated that islet cell autoimmunity may start out as a nonpathogenic Th2 response to the $\beta$ cells that subsequently turns into a pathogenic Th1 response associated with the maturation of the humoral immune response to different antigens in some unfortunate individuals who eventually present with type 1 diabetes $(9-12)$. Th1 immunity is associated with the generation of $\operatorname{IgG} 2 \mathrm{a}$ and $\operatorname{IgG} 3$ in the mouse, whereas a Th2 response mainly results in the generation of $\operatorname{IgG} 1(8,13)$, but the equivalent antibody responses have not been clearly defined in humans. There is some evidence that Th1-dominated T-cell immunity is associated with the generation of interferon- $\gamma$ (INF- $\gamma$ ), tumor necrosis factor- $\alpha$ (TNF- $\alpha$ ), and IL-2 and increased synthesis of IgG1 antibodies, whereas a Th2dominated immune response seems to be associated with subclass $\mathrm{IgG} 4$ and with $\mathrm{IgE}$ antibodies stimulated by the cytokines IL-4 and IL-10 (14-17).

Studies of patients with type 1 diabetes have shown that islet cell antibodies (ICA) are restricted mainly to subclass IgG1 $(18,19)$, and the same holds true for GAD65 antibodies (4, 20-22) and IA-2 antibodies (21). Subclass IgG3 and the IgM and $\operatorname{IgE}$ autoantibody isotypes are also detected in the case of GAD65 and IA-2 but at lower prevalences than $\operatorname{IgG1}(21,22)$. There are also a few studies on the isotype profile of autoantibodies to GAD65 in prediabetic individuals who manifest type 1 diabetes during prospective follow-up and in children who remain unaffected, and one of these suggested a more immature isotype distribution in the nonprogressors who had higher levels of IgE and IgM antibodies than the progressors (9), but conflicting results have also been reported (21). Little is known about the immunoglobulin isotype profile of IAA, but they have been reported to be of either IgG or IgM class (23, 24). In patients with type 1 diabetes, insulin antibodies comprise mainly IgG1 antibodies, whereas IgG3 antibodies are more prevalent before the initiation of exogenous insulin (25). Findings in the offspring of parents with type 1 diabetes have suggested that the IAA isotypes are mostly IgG1 and in a few cases also IgG4, but the presence of the latter was not associated with protection from clinical disease (4).

The aim of this work was to assess the maturation of the humoral immune response to insulin in preclinical type 1 diabetes by observing the emergence of various isotypes of IAA (IgG subclasses and IgA) after the initial appearance of such antibodies in genetically susceptible young children identified from the general population. The analysis of autoantibody isotypes might provide more sensitive and specific markers for discriminating between progression and nonprogression to clinical type 1 diabetes.

\section{METHODS}

Subjects. The series was derived from the Finnish Type 1 Diabetes Prediction and Prevention (DIPP) Study, which is a large ongoing population-based survey of genetically suscep- tible individuals aimed at studying the natural course of preclinical type 1 diabetes and assessing the predictive value of various immune and genetic risk markers in the general population (26). According to the study protocol, newborn infants who carry HLA-DQB1 genotypes conferring susceptibility to type 1 diabetes $[* 02 / * 0302 ; * 0302 / \mathrm{x}(\mathrm{x} \neq * 0301, * 0602$, or $* 0603$ ) and boys who were born in Turku with *02/x] were observed from birth for the appearance of diabetes-associated autoantibodies. Blood samples were obtained at birth and subsequently at intervals of 3-6 mo up to the age of $2 \mathrm{y}$ and after that at intervals of 6-12 mo. ICA were used for primary screening of $\beta$-cell autoimmunity. When a child seroconverted to positivity for ICA, IAA, GAD65Ab, and the protein tyrosine phosphatase-related IA-2 protein (IA-2A) were analyzed in all available samples from birth. Families with a child who persistently tested positive for autoantibodies (positivity in at least two consecutive samples) were invited to take part in a randomized controlled intervention trial to assess whether it is possible to delay or prevent the manifestation of clinical diabetes by daily administration of nasal insulin. The study protocol has been approved by the local Ethics Committees, and the parents of the children have given their written informed consent to participation.

Sixty-one (1.4\%) children of a total of 4269 had tested positive for IAA on at least one occasion by the end of May 2000 , and 20 of these had progressed to overt type 1 diabetes during the period of prospective observation. Two of the 61 infants had transplacentally transferred maternal insulin antibodies, which were not considered in the present analysis. Fifteen of the progressors had an IAA-positive serum sample available from more than one time point, and these were included as cases in the present study. Among the remaining 41 subjects, we identified 30 children who had remained nondiabetic (nonprogressors) and could be matched with the progressors for sex, HLA genotype, and IAA-positive observation time. All 30 nonprogressors tested positive for IAA in at least two available samples. The matching concordance achieved was $90 \%$ for both sex and genotype. IAA of various $\operatorname{IgG}$ subclasses and class IgA were analyzed in the serum samples starting from the time at which IAA were first observed or from the previous sample onward if available. Samples taken after the start of the intervention trial were not included in the present analysis.

The mean age of the 15 progressors at the end of follow-up was $1.72 \mathrm{y}$ (range $0.9-3.0 \mathrm{y}$ ) and at the time of the diagnosis of diabetes was $2.15 \mathrm{y}$ (range $0.9-4.3 \mathrm{y}$ ). Eight $(53.3 \%$ ) of them were boys. The mean age of the 30 nonprogressors (15 boys; $50 \%$ ) at the end of the follow-up was 2.14 y (range $0.8-4.0 ; p=0.06$ ). Five of the 15 progressors carried the high-risk genotype HLA-DQB $1 * 02 / * 0302$, nine had the moderate-risk genotype HLA-DQB $1 * 0302 / \mathrm{x}$, and one boy had the HLA-DQB $1 * 02 / x$ genotype. Among the nonprogressors, 11 of the 30 matched children had the high-risk genotype HLADQB $1 * 02 / 0302$ and 19 carried the moderate-risk genotype HLA-DQB $1 * 0302 / x$. Each child who progressed to clinical type 1 diabetes during the follow-up was observed either up to the diagnosis or up to the inclusion in the intervention trial, if the latter came first, and each nonprogressor up to the sample 
obtained at the corresponding age or up to the inclusion in the intervention trial. There was no difference in the matched observation time between the progressors (mean $1.1 \mathrm{y}$; range $0.4-2.0 \mathrm{y}$ ) and nonprogressors (mean $1.1 \mathrm{y}$; range $0.2-2.0 \mathrm{y} ; p$ $=0.70)$. The number of samples per subject varied from 3 to 7 (median 4 ) in the progressors and from 2 to 7 (median 4 ) in the nonprogressors $(p=0.92)$.

Assays for IAA and their isotypes. Total IAA were analyzed with a radiobinding microassay using $5 \mu \mathrm{L}$ of serum as described previously (27). Immune complexes were precipitated with protein A Sepharose (Pharmacia Biotech, Uppsala, Sweden), and the results were expressed in relative units (RU) based on a standard curve run on each plate. The cut-off limit for IAA positivity (1.56 RU) represents the 99th percentile in 371 Finnish children and adolescents. The intra-assay and interassay coefficients of variation were less than $8 \%$ and less than $12 \%$, respectively. The disease sensitivity of our microassay was $44 \%$ and the specificity was $100 \%$, based on the 2002 Centers for Disease Control and Prevention-sponsored Diabetes Autoantibody Standardization Program workshop. All samples with IAA levels between the 97.5th and 99.5th percentiles were retested to confirm the antibody status. The samples from children seroconverting to IAA negativity were reanalyzed in the same assay run to verify the inverse seroconversion.

Isotype- and subclass-specific IAA were analyzed in an assay based on the same principles as that used for total IAA, the protein A Sepharose precipitation being replaced by monoclonal subclass-specific antibodies linked to streptavidin agarose (Pierce and Warriner, Chester, UK). The volume of serum used per assay was $5 \mu \mathrm{L}$. No competitive inhibition with unlabeled insulin was performed. The biotinylated monoclonal mouse antibodies to human IgG1 (clone G17-1), IgG2 (G1821), IgG3 (G18-3), IgG4 (JDC-14), IgA (G20-359), IgM (G20127), and $\operatorname{IgE}(\mathrm{G} 7-26)$ and to rat $\operatorname{IgM}(\mathrm{G} 53-238)$ were obtained from PharMingen (San Diego, CA, U.S.A.). The streptavidin agarose beads were washed thoroughly with PBS [50 $\mathrm{mM}$ of phosphate buffer, $150 \mathrm{mM}$ of $\mathrm{NaCl}$ ( $\mathrm{pH} \mathrm{7.4)]} \mathrm{before} \mathrm{use,} \mathrm{and}$ the biotinylated antibodies were linked to them by incubating $10 \mu \mathrm{g}$ of antibody per $15 \mu \mathrm{L}$ of beads in $50 \mu \mathrm{L}$ of PBS with vigorous shaking at $4^{\circ} \mathrm{C}$ for $1 \mathrm{~h}$. Thereafter, the beads were washed once with PBS and twice with TBT [50 mM of Tris, $1 \%$ Tween-20 (pH 8.0)]. Finally, $15 \mu \mathrm{L}$ of the beads was suspended in TBT buffer (total volume of suspension $50 \mu \mathrm{L}$ ) and used for the precipitation, which was performed at $4^{\circ} \mathrm{C}$ for $2 \mathrm{~h}$ with vigorous shaking. The results were expressed as SD scores (SDS) calculated from the following equation: SDS = $[\Delta \mathrm{cpm}(=\mathrm{IgG}$ subclass or isotype-specific $\mathrm{cpm}-$ unspecific anti-rat IgM cpm) - mean $\Delta$ cpm of control subjects]/SD $\Delta$ cpm of control samples as described previously (4). Forty-four nondiabetic young Finnish subjects [mean age $9.5 \pm 4.1$ (SD); range $0.6-16.6 \mathrm{y}]$ were used as control subjects. The threshold for positivity was set at $3 \mathrm{SDS}$. This cut-off limit was exceeded by one control subject in the IgG1-IAA (4.38 SDS), IgG3-IAA (3.47 SDS), IgG4-IAA (5.05 SDS), and IgA-IAA (3.27 SDS) assays. All samples from each individual were analyzed in the same assay run. The intra-assay coefficient of variation was less than $18 \%$ in the isotype-specific assays. The method used for the analysis of IAA isotypes is identical to that used for the detection of GAD65Ab isotypes in our laboratory, and the sensitivity and specificity of the method have been tested with IgG1- and IgG3-specific mAb. We have also participated in the first workshop on GAD65Ab isotypes, the results of which were presented at the meeting of the Immunology of Diabetes Society in Chennai, India, in February 2001. Our assay performed well in that workshop. A proportion of both the initially positive and negative samples was retested blindly to confirm the antibody status and titers.

Assays for other diabetes-associated autoantibodies. ICA were quantified by a standard indirect immunofluorescence method on sections of frozen human pancreas from a blood group $\mathrm{O}$ donor (28). The end-point dilution titers of ICApositive samples were recorded, and the results were expressed in Juvenile Diabetes Foundation units. The detection limit was 2.5 Juvenile Diabetes Foundation units. All samples initially positive for ICA were retested to confirm antibody positivity. The sensitivity of the ICA assay in our laboratory was $100 \%$, and the specificity was $98 \%$ in the most relevant international standardization workshop.

The antibodies to GAD65 $\mathrm{Ab}$ and to IA-2A were quantified with specific radiobinding assays as described previously (29, 30 ). The antibody results were expressed in RU based on a standard curve constructed from a dilution of a pool of highly positive samples with a negative sample. The cut-off limit for GAD65 Ab antibody positivity was 5.36 RU and for IA-2A positivity was $0.43 \mathrm{RU}$ (the 99th percentiles for more than 370 nondiabetic Finnish children and adolescents). The disease sensitivity of the GAD65Ab assay was $82 \%$ and that of the IA-2A assay was $62 \%$, based on the 2002 Centers for Disease Control and Prevention-sponsored Diabetes Autoantibody Standardization Program workshop. The corresponding disease specificities were $98 \%$ and $100 \%$. All samples with antibody levels between the 97.5th and 99.5th percentiles were retested to confirm the antibody status.

Data handling and statistical analysis. The unpaired and paired $t$ tests were used to compare mean ages and age differences. The Bonferroni correction for multiple comparisons was performed, where appropriate. The distribution of insulin autoantibody isotypes between the various groups was evaluated by cross-tabulation and $\chi^{2}$ statistics. Individual areas under the curve (AUCs) over the observation period were calculated for total IAA and each isotype, as previously described (31) to avoid the problems associated with multiple data points. The Mann-Whitney $U$ test was used to compare the isotype-specific levels and AUCs between the two groups studied. Correlation analyses were performed with the parametric Pearson test (r) when analyzing age associations and with the nonparametric Spearman test $\left(\mathrm{r}_{\mathrm{s}}\right)$ in the other correlation analyses. A two-tailed $p=0.05$ or less was considered to be statistically significant.

\section{RESULTS}

Positivity for autoantibodies. As defined by the inclusion criteria, all study children were positive for IAA or some IAA isotype on inclusion in the series. ICA were initially observed in eight $(53 \%)$ of the 15 progressors and in eight $(27 \%)$ of the 
30 nonprogressors, GAD65 $\mathrm{Ab}$ in five $(33 \%)$ progressors and eight $(27 \%)$ nonprogressors, and IA-2A in two progressors. Positivity for three or more antibodies was found initially in four $(27 \%)$ progressors and two $(7 \%)$ nonprogressors $(p=$ 0.06 ). Seroconversion to IAA positivity occurred at the age of $1.04 \mathrm{y}$ (range $0.3-2.2 \mathrm{y}$ ) among the progressors and at the age of $1.40 \mathrm{y}$ (range $0.6-3.6 \mathrm{y} ; p=0.08$ ) among the nonprogressors, the median levels of initial IAA being $10.05 \mathrm{RU}$ (range 1.7-84.4 RU) and 6.00 RU (range 1.6-46.8 RU; $p=0.44$ ), respectively. IAA remained positive and relatively stable after their emergence in the progressors, but inverse seroconversions were seen in seven of the nonprogressors $(p=0.04$ relative to progressors).

Appearance of IAA isotypes in the total series. IgG1-IAA appeared in 43 children at a mean age of $1.30 \mathrm{y}$ (range 0.2-4.0 y), followed by IgG3-IAA in 33 at a mean age of 1.31 y (range $0.5-3.6 \mathrm{y}$ ) and IgG2-IAA in 25 at a mean age of $1.35 \mathrm{y}$ (range $0.3-2.5 \mathrm{y}$ ). IgG4-IAA emerged at a mean age of $1.56 \mathrm{y}$ (range $1.0-2.5 \mathrm{y}$ ) in 15 children and IgA at a mean age of $1.2 \mathrm{y}$ (range $0.2-3.6$ y) in eight children. IgG1-IAA $(p<0.001)$ and $\operatorname{IgG2-IAA}(p=0.01)$ appeared earlier than IgG4-IAA in terms of pairwise comparisons between the isotypes.

Distribution of IAA isotypes in the initial sample. The frequencies of the various isotypes in initial IAA-positive samples are shown in Figure 1A, and the corresponding titers in SDS are shown in Figure $2 A$. IgG3-IAA were detected more frequently in the progressors than in the nonprogressors. There were no significant differences in the titers of isotype-specific antibodies in the initial sample. The number of isotypes detectable in the first positive sample was significantly higher among the progressors (median 2) than among the nonprogressors (median $1 ; p=0.003$ ).

IgG subclass changes during follow-up. All of the progressors experienced seroconversion to positivity for additional isotypes, and accordingly they all had both detectable IgG1IAA and IgG3-IAA at least once. IgG2, IgG4, and IgA-IAA were also seen in 10, six, and five subjects, respectively, during the observation period, and one child had isotype levels fluctuating from positivity to negativity and back to positivity. Inverse seroconversions occurred in six children, three of whom had initially tested positive for IgG3. IgG1 was the most consistent isotype. Positive seroconversions were also seen among the nonprogressors but not as often. IgG1-IAA (Fig. $1 B$ ) were observed in 28 of the 30 nonprogressors, IgG3-IAA in $18,(p=0.004$ versus progressors), IgG2-IAA in 15 , IgG4IAA in nine, and IgA-IAA in three ( $p=0.05$ versus progressors). Both IgG1 and IgG3-IAA appeared earlier in the progressors than in the nonprogressors (Table 1). There was also a correlation between the age of appearance of total IAA and the ages of appearance of the various isotypes. The closest correlation among the progressors was seen between IgG3IAA and IAA $(r=0.96 ; p<0.001)$ and secondly between IgG1-IAA and IAA $(r=0.66 ; p=0.008)$, whereas among the nonprogressors, correlations were observed between IgG3IAA and IAA ( $r=0.95 ; p<0.001)$, IgG1-IAA and IAA $(r=$ 0.94; $p<0.001)$, IgG4-IAA and IAA $(r=0.88 ; p=0.002)$, and IgG2-IAA and IAA $(r=0.63 ; p=0.01)$. The progressors had higher maximum levels of IgG3-IAA during the follow-up

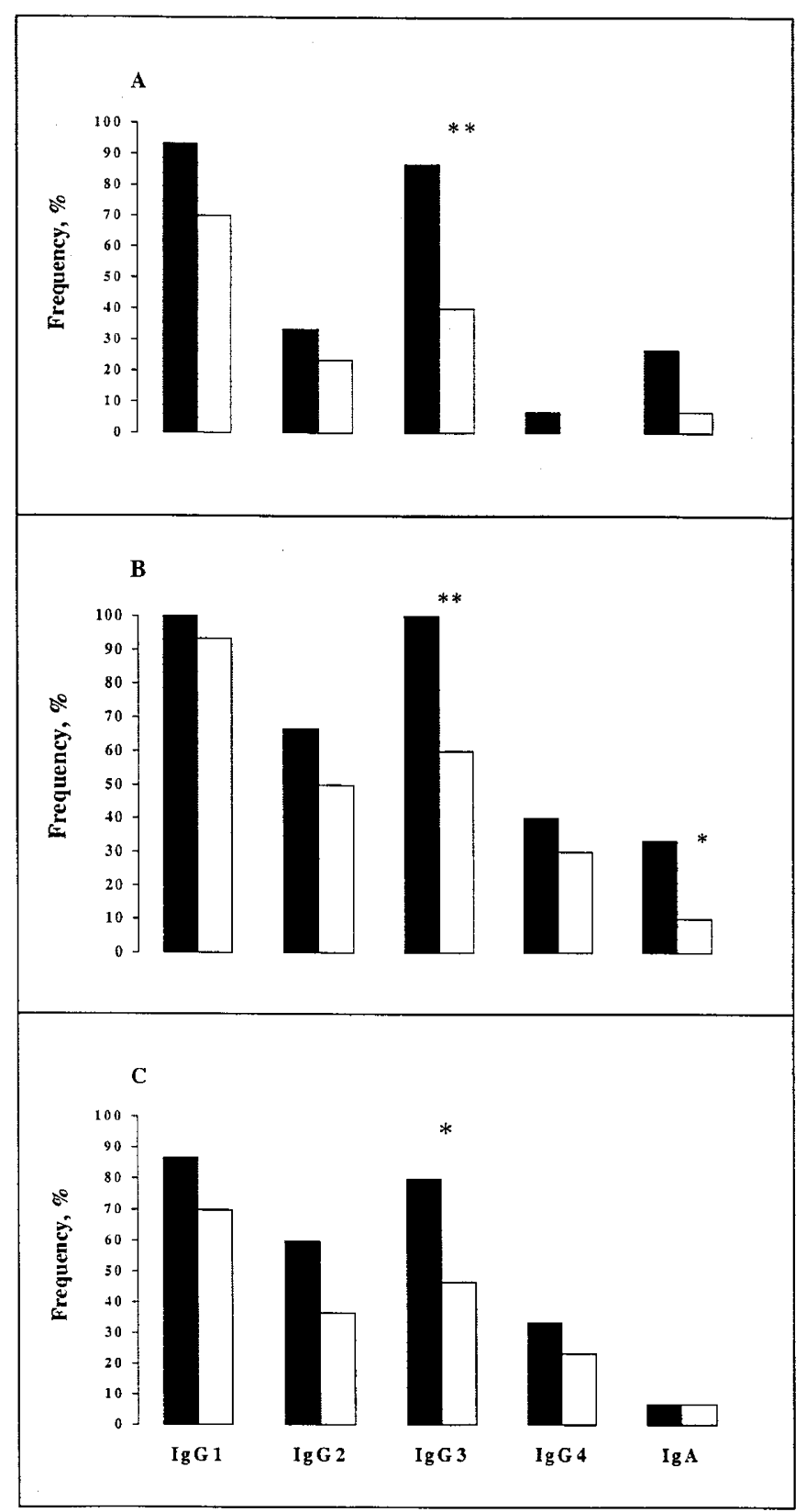

Figure 1. Frequencies of IAA subclasses in the samples collected from the study children during prospective follow-up in 15 progressors ( $\square$ ) and 30 nonprogressors $(\square)$. The findings in the first IAA-positive sample $(A)$, in the follow-up sample containing the maximum number of isotypes $(B)$, and in the last sample analyzed $(C) ;{ }^{*} p \leq 0.05,{ }^{* *} p=0.01$ ( $\chi^{2}$ statistics $)$.

than the nonprogressors (Fig. $2 B$ ). The maximum number of detectable isotypes during the follow-up was higher in the progressors (median 4) than in the nonprogressors (median 3; $p=0.02$ ). The only significant difference in the isotype frequencies between the two groups in the last sample analyzed was for IgG3-IAA, which was detected in 12 progressors and in 14 nonprogressors $(p=0.03$; Fig. $1 C)$. In the last sample, higher titers of IgG1-IAA, IgG3-IAA, and IgA-IAA were seen in the progressors than in the nonprogressors (Fig. 2C), who had a lower number of detectable isotypes (median 2) than the progressors (median $4 ; p=0.01$ ). When comparing the proportion of positive children between the initial sample and the 


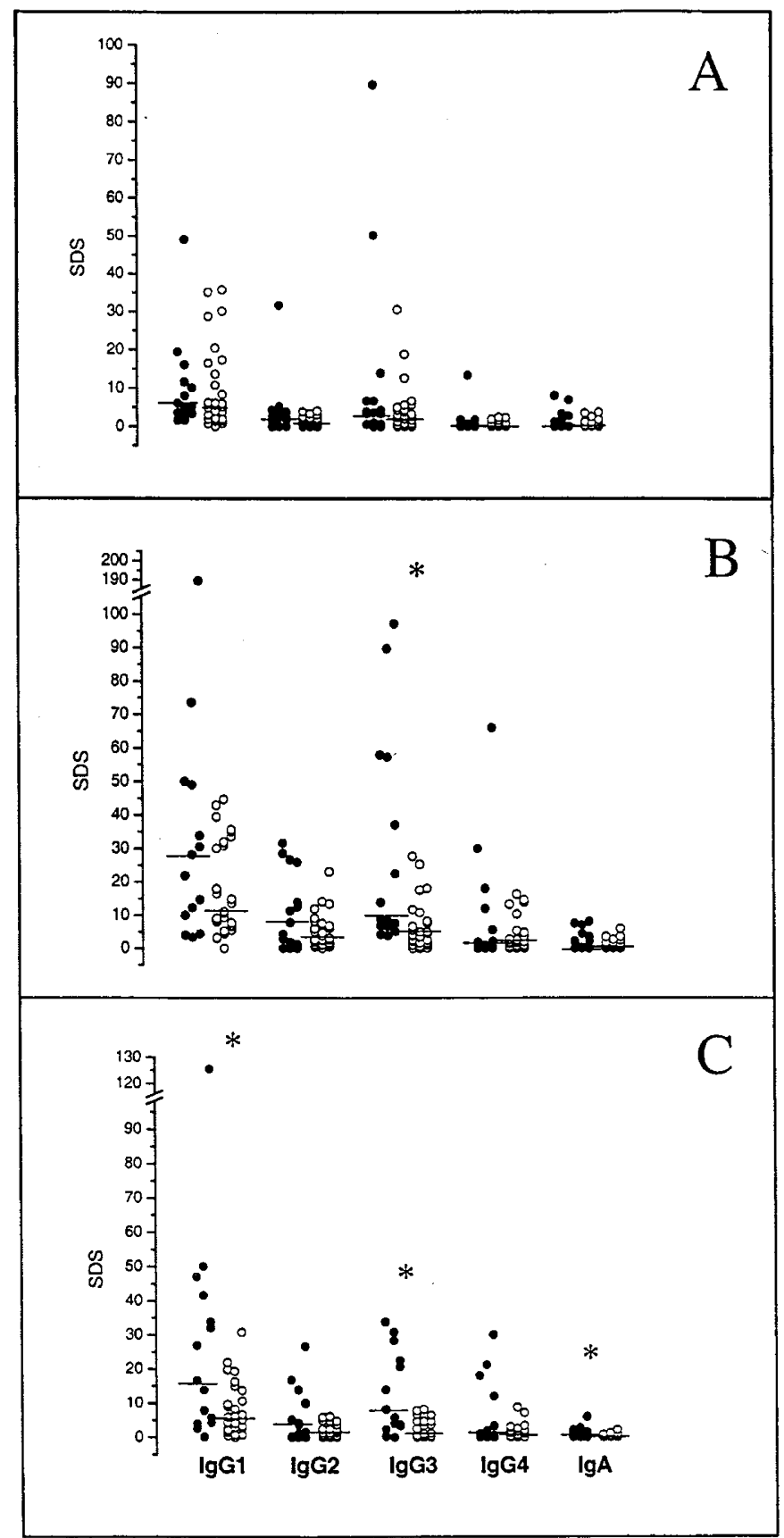

Figure 2. Titers (SDS) of IAA subclasses in the samples collected from the study children during prospective follow-up in 15 progressors $(\bullet)$ and 30 nonprogressors $(\bigcirc)$. The findings in the first IAA-positive sample $(A)$, in the follow-up sample containing the maximum isotype levels $(B)$, and in the last sample analyzed $(C)$. The lines represent median values; ${ }^{*} p<0.05$ (MannWhitney $U$ test).

sample with the maximum number of isotypes, a significant isotype spreading was observed for IgG4-IAA $(p=0.05)$ among the progressors. Isotype spreading was also frequent among the nonprogressors and was observed to be significant for IgG1-IAA $(p=0.006)$, IgG2-IAA $(p=0.03)$, and IgG4IAA $(p=0.001)$. There were correlations between the level of total IAA and the number of isotypes in the first positive sample $\left(\mathrm{r}_{\mathrm{s}}=0.64 ; p<0.01\right)$, in the sample with the maximum
Table 1. Mean age [years (range)] at the appearance of IAA and IAA isotypes in 15 progressors and 30 nonprogressors

\begin{tabular}{llll}
\hline & \multicolumn{1}{c}{$\begin{array}{c}\text { Progressors } \\
(n=15)\end{array}$} & \multicolumn{1}{c}{$\begin{array}{c}\text { Nonprogressors } \\
(n=30)\end{array}$} & $p$ value \\
\hline IAA & $1.04(0.3-2.2 ; n=15)$ & $1.40(0.6-3.6 ; n=30)$ & 0.08 \\
IgG1-IAA & $0.91(0.2-1.6 ; n=15)$ & $1.51(0.3-4.0 ; n=28)$ & 0.003 \\
IgG2-IAA & $1.20(0.3-1.9 ; n=10)$ & $1.45(0.4-2.5 ; n=15)$ & 0.26 \\
IgG3-IAA & $1.09(0.5-2.2 ; n=15)$ & $1.50(0.6-3.6 ; n=18)$ & 0.05 \\
IgG4-IAA & $1.40(1.0-2.0 ; n=6)$ & $1.70(1.0-2.5 ; n=9)$ & 0.19 \\
IgA-IAA & $0.68(0.2-1.6 ; n=5)$ & $2.11(0.99-3.6 ; n=3)$ & 0.07 \\
\hline
\end{tabular}

number of isotypes $\left(\mathrm{r}_{\mathrm{s}}=0.60 ; p<0.01\right)$, and in the last available sample $\left(\mathrm{r}_{\mathrm{s}}=0.81 ; p<0.01\right)$.

Dominant isotype responses. Nine progressors had a dominant IgG1-IAA response initially and six had a dominant initial IgG3-IAA response, whereas the corresponding distribution among the nonprogressors was 20/0/3 (three other than IgG1- or IgG3-IAA; $\left.\chi_{\mathrm{df}=2}^{2}=12.02 ; p=0.002\right)$. On calculating the AUC, we observed IgG1-IAA dominance during the follow-up period in nine (60\%) progressors and $22(73 \%)$ nonprogressors. IgG3-IAA dominance was seen in six $(40 \%)$ progressors but in only one (3\%) nonprogressor. IgG2-IAA dominated in six (20\%) nonprogressors, and one nonprogressor had a dominant IgA-IAA response. The distribution of various dominant isotypes between the progressors and nonprogressors was significantly different $\left(\chi_{\mathrm{df}=2}^{2}=11.23 ; p=0.004\right)$. The profiles of total IAA and various IAA isotypes in the six progressors with a dominant $\operatorname{IgG} 3$ response are shown in Figure 3 in relation to the follow-up time. The pattern was similar when relating the integrated isotype levels to total IAA. Most progressors (60\%) had IgG1-IAA dominance, and the remaining ones had IgG3-IAA dominance. Using median values, we noticed the rank order of the isotypes to be $\operatorname{IgG} 1>\operatorname{IgG} 3>\operatorname{IgG} 2>\operatorname{IgG} 4$ at the beginning and to remain the same during observation in the progressors, whereas the non-

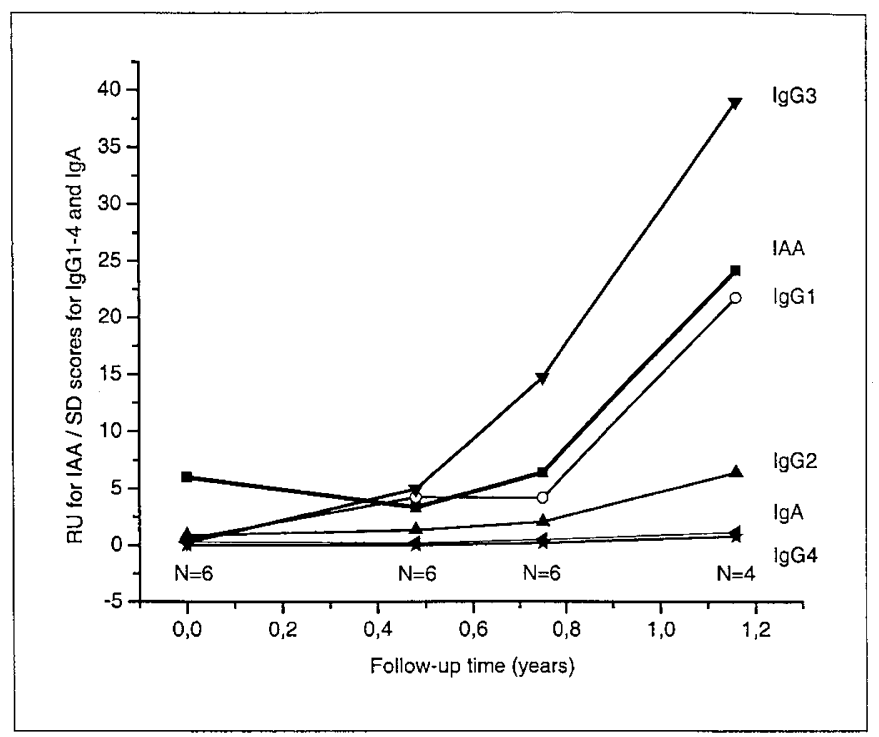

Figure 3. The profile of total IAA and IAA isotypes as a function of the follow-up time in children $(n=6)$ with a dominant IgG3-IAA response. The values are medians, and the time points for sampling are shown based on the means in the six children. 
progressors had the isotypes in identical order at the beginning (IgG1 $>\operatorname{IgG} 3>\operatorname{IgG} 2>\operatorname{IgG} 4)$, but their median levels of IgG3, $\mathrm{IgG} 2$ and IgG4 were markedly lower than in the progressors and the isotype order changed to $\operatorname{IgG} 1>\operatorname{IgG} 2>\operatorname{IgG} 3>\operatorname{IgG} 4$ during the follow-up.

The progressors had significantly higher levels of IgG1-IAA $(p=0.05)$ and IgG3-IAA $(p=0.002)$ based on AUC (Table $2)$, and the IgG3-IAA AUC remained significantly higher in the progressors $(p=0.04)$ when comparing relative levels. There were no significant differences in the integrated levels of IgG2, IgG4, or IgA-IAA between the two groups. On dividing the groups of progressors and nonprogressors into subgroups according to their total IAA levels (above or below the median IAA level of $16.5 \mathrm{RU}$ ), we observed that the progressors with high IAA levels also tended to have higher integrated levels of $\operatorname{IgG2}-\mathrm{IAA}(p=0.07)$ than the corresponding nonprogressors. Sex, HLA DQB1 genotype, and total number of autoantibodies had no effect on the integrated values.

\section{DISCUSSION}

Given that the isotype profile of antigen-specific autoantibodies may reflect either a Th1 or a Th2 immune response, the aim here was to assess the maturation of the humoral immune response to insulin in preclinical type 1 diabetes by observing the emergence of various isotypes of IAA after the initial appearance of such antibodies in genetically susceptible children identified from the general population. We observed that the number of detectable isotypes was higher and more stable among those who progressed to clinical type 1 diabetes. There was some heterogeneity in the IAA isotype response, but most frequently an IgG1-IAA response was the first to appear, whereas IgG4-IAA appeared late.

The present series is unique, because the observation started before or at the time of seroconversion to IAA positivity with sequential sampling with an interval of 3-6 mo. The children studied were grouped into progressors and nonprogressors on the basis of whether they presented with type 1 diabetes or not during the observation period. Admittedly, this classification

Table 2. Absolute integrated levels of IAA and IAA isotypes and relative (total IAA-corrected) AUC values of IAA isotypes over the observation time in 15 progressors and 30 nonprogressors based on AUC analysis

\begin{tabular}{lcll}
\hline & $\begin{array}{c}\text { Progressors } \\
(n=15)\end{array}$ & $\begin{array}{c}\text { Nonprogressors } \\
(n=30)\end{array}$ & $p$ value \\
\hline Absolute AUC values & & & \\
IAA & $18.9 ; 4.6-40.2$ & $7.3 ; 3.9-17.0$ & 0.07 \\
IgG1-IAA & $17.0 ; 8.3-28.2$ & $6.5 ; 4.3-15.9$ & 0.05 \\
IgG2-IAA & $4.1 ; 0.8-10.5$ & $1.7 ; 0.6-4.2$ & 0.15 \\
IgG3-IAA & $7.0 ; 2.8-23.0$ & $1.7 ; 0.6-3.9$ & 0.002 \\
IgG4-IAA & $0.6 ; 0.1-6.1$ & $0.8 ; 0.1-2.8$ & 0.95 \\
IgA-IAA & $0.2 ; 0.1-3.6$ & $0.4 ; 0.0-0.6$ & 0.37 \\
Relative levels & & & \\
IgG1-IAA/total IAA & $0.89 ; 0.72-1.20$ & $0.92 ; 0.72-1.10$ & 0.98 \\
IgG2-IAA/total IAA & $0.14 ; 0.08-0.44$ & $0.21 ; 0.08-0.33$ & 0.77 \\
IgG3-IAA/total IAA & $0.53 ; 0.21-1.12$ & $0.28 ; 0.15-0.49$ & 0.04 \\
IgG4-IAA/total IAA & $0.02 ; 0.00-0.08$ & $0.07 ; 0.02-0.21$ & 0.17 \\
IgG5-IAA/total IAA & $0.03 ; 0.00-0.13$ & $0.03 ; 0.00-0.11$ & 0.99 \\
\hline
\end{tabular}

Data are medians; interquartile ranges. does not exclude the possibility that some of those classified as "nonprogressors" may later manifest type 1 diabetes. Accordingly, the group of progressors represents those who presented with clinical diabetes at a very young age. More than $90 \%$ of the progressors and two thirds of the nonprogressors had an IgG1-IAA response in their first IAA-positive sample. The initial response more often also included IgG2, IgG3, IgG4, and IgA-IAA in the case of the progressors than in the nonprogressors. The initial IAA response was characterized by a dominant peak, which comprised IgG1 in nine progressors and in 20 nonprogressors, the remaining six progressors having a dominant IgG3-IAA response, whereas IgG3 dominance was not seen initially among the nonprogressors. Previous studies of the IgG subclasses of ICA, GAD65, and IA-2 in patients with newly diagnosed diabetes and first-degree relatives progressing to diabetes have shown a similar IgG1 dominance (4, $18-21,31-33)$.

All of the progressors had IgG1- and IgG3-IAA responses during prospective observation, whereas the IgG3-IAA response in particular was less frequent in the nonprogressors. Upon calculating the integrated levels (AUC), we observed IgG1-IAA dominance in approximately half of the progressors and in close to $75 \%$ of the nonprogressors, whereas IgG3-IAA dominance was seen in six (40\%) of the progressors but in only one $(3 \%)$ nonprogressor. Comparison of the isotype proportions in relation to total IAA yielded a similar pattern, two thirds of the progressors having IgG1 dominance and the remainder having IgG3 dominance. The relative AUC for IgG3-IAA remained significantly increased in the progressors, excluding the possibility that the observed difference was only a consequence of the fact that the progressors tended to have higher levels of total IAA than the nonprogressors. The rank order of $\operatorname{IgG} 3$ decreased in the nonprogressors from position 2 to position 3 among the IgG subclasses during the follow-up, whereas the rank order remained unchanged in position 2 among the progressors. These observations suggest that a weak or absent IgG3-IAA response could be a protective sign in IAA-positive individuals. No particular isotype switching pattern was seen during the follow-up, but positive seroconversions were more frequent among the progressors and the isotypes were more stable. Samples taken after randomization to the controlled intervention trial of the DIPP study with intranasal insulin were not included in the present analysis, as treatment with intranasal insulin may potentially have on effect on the isotype profile of IAA. Such data, however, cannot be generated before the codes of the intervention trial are broken.

Approximately half of both the progressors and the nonprogressors developed a low IgG4-IAA response during the follow-up period, indicating that IgG4-IAA cannot discriminate nonprogressors from progressors. This observation is consistent with the finding in offspring of parents who are affected by type 1 diabetes in the German BABYDIAB study (4). Contrasting results supporting the hypothesis that IgG4 isotypes reflect a Th2 response have been reported for GAD65 $\mathrm{Ab}$ in one paper, where IgG4 and/or IgG2 was associated with nonprogression to disease in older first-degree relatives (20), but in that case, the detection was based on the ELISA technique. 
The present data show that genetically susceptible young children who progress to clinical type 1 diabetes are characterized by strong IgG1 and IgG3 responses to insulin, indicating a powerful insulin-specific Th1 response. A weak IgG3IAA response or the total lack of such a response is associated with relative protection against clinical disease, because one third of the nonprogressors had no detectable $\operatorname{IgG} 3$ response during follow-up and the IgG3 response was dominant in only one nonprogressor. This indicates that nonprogressors are characterized by a weak IgG3 response to insulin, possibly reflecting attenuated insulin-specific Th1 reactivity.

Acknowledgments. We are indebted to Tuula Simell, Maija Törmä, Birgitta Nurmi, Hilkka Pohjola, Aino Stenius, Kaisu Riikonen, Ulla Markkanen, Paula Asunta, Aila Suutari, and Riikka Sihvo for commitment to the study. We are also grateful to Riitta Päkkilä, Susanna Heikkilä, Päivi Salmijärvi, Tuovi Mehtälä, Sirpa Anttila, and Sirpa Pohjola for skillful technical assistance.

\section{REFERENCES}

1. Castano LE, Eisenbarth GS 1990 Type 1 diabetes: a chronic autoimmune disease of man, mouse and rat. Annu Rev Immunol 8:647-679

2. Palmer JP 1993 Predicting type 1 diabetes. Use of humoral immune markers. Diabetes Rev 1:104-115

3. Atkinson MA, Maclaren NK, Riley WJ, Winter WE, Fisk DD, Spillar RP 1986 Are insulin autoantibodies markers for insulin-dependent diabetes mellitus? Diabetes 35:894-898

4. Bonifacio E, Scirpoli M, Kredel K, Fuchtenbusch M, Ziegler AG 1999 Early autoantibody responses in prediabetes are IgG1 dominated and suggest antigenspecific regulation. J Immunol 163:525-532

5. Kimpimäki T, Kupila A, Hämäläinen AM, Kukko M, Kulmala P, Savola K, Simell T, Keskinen P, Ilonen J, Simell O, Knip M 2001 The first signs of $\beta$-cell autoimmunity appear in infancy in genetically susceptible children from the general population: the Finnish Type 1 Diabetes Prediction and Prevention study. J Clin Endocrinol Metab $86: 4782-4788$

6. Karjalainen J, Knip M, Mustonen A, Ilonen J, Åkerblom HK 1986 Relation between insulin antibody and complement fixing islet cell antibody at clinical diagnosis of IDDM. Diabetes 35:620-622

7. Vardi P, Ziegler AG, Mathews JH, Dib S, Keller RJ, Ricker AT, Wolfsdorf JI, Herskowitz RD, Rabizadeh A, Eisenbarth GS 1988 Concentration of insulin autoantibodies at onset of type 1 diabetes. Inverse log-linear correlation with age. Diabetes Care 11:736-739

8. Toellner KM, Luther SA, Sze DM, Choy RK, Taylor DR, MacLennan IC, AchaOrbea H 1998 T helper 1 (Th1) and Th2 characteristics start to develop during T cell priming and are associated with an immediate ability to induce immunoglobulin class switching. J Exp Med 187:1193-1204

9. Petersen JS, Kulmala P, Clausen JT, Knip M, Dyrberg T, the Childhood Diabetes in Finland Study Group 1999 Progression to type 1 diabetes is associated with a change in the immunoglobulin isotype profile of autoantibodies to glutamic acid decarboxylase. Clin Immunol 2:276-281

10. Katz JD, Benoist C, Mathis D $1995 \mathrm{~T}$ helper cell subsets in insulin-dependen diabetes. Science 26:1185-1188
11. Pilström B, Björk L, Böhme J 1995 Demonstration of Th1 cytokine profile in the late phase of NOD insulitis. Cytokine 8:806-814

12. Nicholson LB, Kuchroo VK 1996 Manipulation of the Th1/Th2 balance in autoimmune disease. Curr Opin Immunol 8:837-842

13. Abbas AK, Murphy KM, Sher A 1996 Functional diversity of helper T lymphocytes. Nature 383:787-793

14. Kallmann BA, Huther M, Tubes M, Feldkamp J, Bertrams J, Gries FA, Lampeter EF, Kolb H 1997 Systemic bias of cytokine production toward cell-mediated immune regulation in IDDM and toward humoral immunity in Graves' disease. Diabetes 46:237-243

15. Ng WY, Thai AC, Lui KF, Yeo PP, Cheah JS 1999 Systemic levels of cytokine and GAD-specific autoantibody isotypes in Chinese IDDM patients. Diabetes Res Clin Pract 43:127-135

16. Lundgren M, Persson U, Larsson P, Magnusson C, Smith CI, Hammarstrom L, Severinson E 1989 Interleukin 4 induces synthesis of $\mathrm{IgE}$ and IgG4 in human B cells. Eur J Immunol 7:1311-1315

17. Kallmann BA, Lampeter EF, Hanifi-Moghaddam P, Hawa M, Leslie RDS, Kolb H 1999 Cytokine secretion patterns in twins discordant for type 1 diabetes. Diabetologia 42:1080-1085

18. Dozio N, Belloni C, Girardi AM, Genovese S, Sodoyez JC, Bottazzo GF, Pozza G, Bosi E 1994 Heterogeneous IgG subclass distribution of islet cell antibodies. J Autoimmun 7:45-53

19. Millward A, Hussain MJ, Peakman M, Pyke DA, Leslie RD, Vergani D 1988 Characterization of islet cell antibody in insulin-dependent diabetes: evidence for IgG1 subclass restriction and polyclonality. Clin Exp Immunol 71:353-356

20. Couper JJ, Harrison LC, Aldis JJE, Colman P, Honeyman MC, Ferrante A 1998 IgG subclass antibodies to glutamic acid decarboxylase and risk for progression to clinical insulin-dependent diabetes. Hum Immunol 59:493-499

21. Hawa MI, Fava D, Medici F, Deng YJ, Notkins AL, De Mattia G, Leslie RD 2000 Antibodies to IA-2 and GAD65 in type 1 and type 2 diabetes: isotype restriction and polyclonality. Diabetes Care 23:228-233

22. Lohmann T, Hawa M, Leslie RD, Lane R, Picard J, Londei M 2000 Immune reactivity to glutamic acid decarboxylase 65 in stiff man syndrome and type 1 diabetes mellitus. Lancet 356:31-35

23. Dean BM, Becker F, McNally JM, Tarn AC, Schwartz G, Gale EA, Bottazzo GF 1986 Insulin autoantibodies in the pre-diabetic period: correlation with islet cell antibodies and development of diabetes. Diabetologia 29:339-342

24. Bodansky HJ, Grant PJ, Dean BM, McNally J, Bottazzo GF, Hambling MH, Wales JK 1986 Islet-cell antibodies and insulin autoantibodies in association with common viral infections. Lancet 2:1351-1353

25. Potter KN, Wilkin TJ 2000 The molecular specificity of insulin autoantibodies. Diabetes Metab Res Rev 16:338-353

26. Kupila A, Muona P, Simell T, Arvilommi P, Savolainen H, Hämäläinen AM, Korhonen S, Kimpimäki T, Sjoroos M, Ilonen J, Knip M, Simell O 2001 Feasibility of genetic and immunological prediction of type 1 diabetes in a population-based birth cohort. Diabetologia 44:290-297

27. Ronkainen M, Hämäläinen AM, Koskela P, Åkerblom HK, Knip M, the Finnish TRIGR Study Group 2001 Pregnancy induces non-immunoglobulin insulin-binding activity in both maternal and cord blood serum. Clin Exp Immunol 124:190-196

28. Bottazzo GF, Florin-Christensen A, Doniach D 1974 Islet-cell antibodies in diabetes mellitus with autoimmune polyendocrine deficiencies. Lancet 2:1279-1282

29. Savola K, Sabbah E, Kulmala P, Vähäsalo P, Ilonen J, Knip M 1998 Autoantibodies associated with type 1 diabetes mellitus persist after diagnosis in children. Diabetologia 41:1293-1297

30. Savola K, Bonifacio E, Sabbah E, Kulmala P, Vähäsalo P, Karjalainen J, TuomilehtoWolf E, Meriläinen J, Åkerblom HK, Knip M 1998 IA-2 antibodies-a sensitive marker of IDDM with clinical onset in childhood and adolescence. Diabetologia 41:424-429

31. Matthews JNS, Altman DG, Campbell MJ, Royston P 1990 Analysis of serial measurements in medical research. BMJ 300:230-235

32. Bruining GJ, Molenaar J, Tuk CW, Lindeman J, Bruining HA, Marner B 1984 Clinical time-course and characteristics of islet cell cytoplasmatic antibodies in childhood diabetes. Diabetologia 26:24-29

33. Schatz DA, Barrett DJ, Maclaren NK, Riley WJ 1988 Polyclonal nature of islet cell antibodies in insulin-dependent diabetes. Autoimmunity 1:45-50 